\title{
Determination of loci associated with potato starch resistivity to hydrolysis by $\alpha$-amylase
}

\author{
Gvozdeva L.M. ${ }^{1 *}$, Rozanova I.V. ${ }^{1,2}$, Khlestkin V.K. ${ }^{1,3}$, Khlestkina E.K. ${ }^{1,2}$ \\ ${ }^{1}$ Institute of Cytology and Genetics, SB RAS, Novosibirsk, Russia \\ ${ }^{2}$ N.I. Vavilov All-Russian Institute of Plant Genetic Resources (VIR), St. Petersburg, Russia \\ ${ }^{3}$ All-Russia Research Institute of Farm Animal Genetics and Breeding - Branch of the L.K. Ernst Federal \\ Science Center for Animal Husbandry, St. Petersburg, Russia \\ *e-mail:ungersy@mail.ru
}

Natural production of raw potato starch with pre-designed physical and chemical properties is a challenging task, which appears to be important for both its industrial biotechnological processing and developing diets for weight reduction as well as for maintenance/treatment and prevention of some diseases (diabetes, obesity, etc.). Resistant starch is considered as important dietary fiber for the nutrition of beneficial bacteria in the large intestine. For the development of potato cultivars with desired starch digestibility the next generation breeding techniques are required. Application of these techniques is based on the information about certain genetic mechanisms, underpinning desired properties. The purpose of this work was to identify the genomic SNP loci associated with resistance and digestibility of potato starch. 90 potato varieties and hybrids from the ICG "GenAgro" collection were genotyped on the Illumina 22K SNP potato array. Raw tuber starch from the same varieties and hybrids samples were isolated and tested for resistance to digestion by $\alpha$-amylase. Contrast forms with low (40-52\%) and high (up to $99 \%)$ resistance were revealed. These data were used for further genome-wide analysis of genotype-phenotype associations (GWAS). Tassel 5 software and the R package were used for analysis. Using the GLM (Generalized Linear Model), a significant association of resistance of tuber starch to amylase digestion was found with the genomic region assigned to chromosome 5 . Based on the results we plan to convert significant SNP to the convenient CAPs marker for further validation and application for marker-assisted selection of plants with different tuber starch digestibility. Acknowledgements: The study is supported by the RFBR (17-29-08006). 